\title{
Peculiar Current Solar-Minimum Structure of the Heliosphere
}

\author{
P. K. Manoharan \\ Radio Astronomy Centre, National Centre for Radio Astrophysics, \\ Tata Institute of Fundamental Research, Udhagamandalam (Ooty), 643001, India \\ email: mano@ncra.tifr.res.in
}

\begin{abstract}
In this paper, I review the results of 3-D evolution of the inner heliosphere over the solar cycle 23, based on observations of interplanetary scintillation (IPS) made at $327 \mathrm{MHz}$ using the Ooty Radio Telescope. The large-scale features of solar wind speed and density turbulence of the current minimum are remarkably different from that of the previous cycle. The results on the solar wind density turbulence show that (1) the current solar minimum is experiencing a low level of coronal density turbulence, to a present value of $\sim 50 \%$ lower than the previous similar phase, and (2) the scattering diameter of the corona has decreased steadily after the year 2003. The results on solar wind speed are consistent with the magnetic field strength at the poles and the warping of heliospheric current sheet.
\end{abstract}

Keywords. turbulence, scattering, Sun: corona, Sun: magnetic fields, Sun: coronal mass ejections (CMEs), solar wind, solar-terrestrial relations

\section{Interplanetary Scintillation}

In this study, a large amount of interplanetary scintillation (IPS) data obtained from the Ooty Radio Telescope (ORT), operating at $327 \mathrm{MHz}$ (Swarup et al. 1971), has been employed to study the 3-D evolution of the heliosphere over the period 1989-2009. The IPS observations made with the ORT can provide the velocity of the solar wind and the scintillation index $(m)$ in the heliocentric distance range of $R \sim 10-250$ solar radii $\left(R_{\odot}\right)$ and at all heliographic latitudes. The value of $m$ is a measure of electron-density turbulence in the solar wind $\left(m^{2} \sim \int \delta N_{e}^{2}(z) d z\right)$, along the line of sight $(z)$ to the radio source (e.g., Manoharan et al. 2000). The normalized scintillation index, $g=m(R) /<m(R)>$ (i.e., observed index normalized by its long-term average) enables the comparison of levels of density turbulence obtained from different sources. However, the value of $g$ is linearly related to $\delta N_{e}$ only in the weak-scattering region at distances at $>40 R_{\odot}$. For example, an $m$ - $R$ profile attains the peak value at the strong-to-weak scattering transition point, which typically occurs $\sim 40 R_{\odot}$ for IPS at $327 \mathrm{MHz}$ (e.g., Manoharan 1993; 2006). In this study, the solar wind velocity and turbulence images have been exclusively obtained from weak-scattering data. However, the contour of constant level of turbulence in a year at different latitudes has been determined using peaks of several $m$ - $R$ profiles.

\section{Solar Cycle 23: Three-Dimensional Solar Wind}

Figure 1a shows the latitudinal distributions of solar wind speed and density turbulence $(g)$ observed at Ooty over the solar cycle 23. These plots are similar to the well-known 'butterfly diagram' of photospheric magnetic field intensity. They have been made by tracing backward/forward from the measurement location onto a sphere of radius $\sim 100$ $\mathrm{R}_{\odot}$, which approximately corresponds to the mid range of distances covered in the observations utilized to generate the plots. 

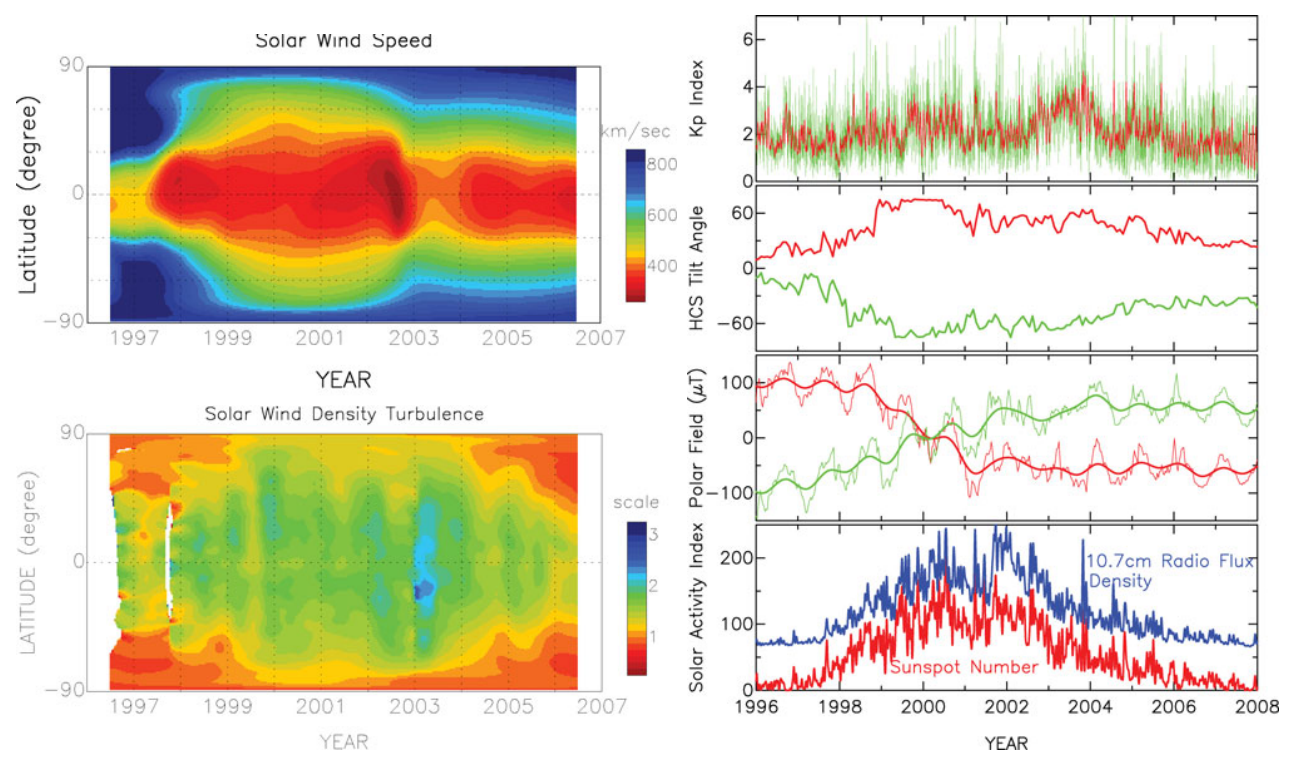

Figure 1. (a) Latitudinal distributions of solar wind speed (left, top) and density turbulence (left, bottom). The contour levels drawn on the speed gray-scale image are $350,450,550$, and 650 $\mathrm{kms}^{-1}$. (b) The stack plot (right) shows geo-magnetic disturbance index, Kp, intensity of polar magnetic field, tilt angle of heliospheric current sheet (HCS), and solar activities (i.e., sunspot number and solar radio flux density at $10.7 \mathrm{~cm}$ ).

It is evident in the 'latitude-year' speed plot that during minimum of the solar cycle, polar regions are dominated by high speed streams $\left(\sim 600-800 \mathrm{kms}^{-1}\right)$ from open-field coronal holes, and low and variable flow speeds $\left(\leqslant 500 \mathrm{kms}^{-1}\right)$ are observed at the lowand mid-latitude regions of the complex/closed field corona. But, there are marked differences in latitudinal extents of low- and high-speed flow regions between the current and previous minimum phases. For example during 1996-97, the low-speed flow was confined to $\sim \pm 30^{\circ}$ of the equatorial belt; whereas at the current minimum, it extends to a latitude range of $\sim \pm 50^{\circ}$. These low-speed wind widths also correlate with the latitudinal warping (tilt angle) of the heliospheric current sheet (HCS) over a small amplitude, $\sim \pm 15^{\circ}$, at the previous minimum and a moderate amplitude, $\sim \pm 30^{\circ}$, at the current minimum, respectively (Figure 1b). These results suggest (i) a near-dipole magnetic field of the Sun for the previous minimum, around the year 1997 and (ii) a never-approached dipole-field geometry during the current minimum. It is in good agreement with the result from other independent IPS speed measurements obtained from the Solar-Terrestrial Environment Laboratory (e.g., Tokumaru et al. 2009). Thus, the HCS tilt of the corona of the current phase tends to resemble a condition similar to that of moderate activity, but without activity!

These changes in the latitudinal extents of low-speed wind have also influenced the high-speed flows from the polar regions. In the current minimum phase, high-speed regions at the poles have remarkably shrunk towards the poles (Figure 1a, top). Moreover, the speed of the high-latitude $\left(>50^{\circ}\right)$ wind is considerably less for the current minimum than for the previous minimum. These findings nicely correlate with the polar field strength, which is $\sim 40-50 \%$ weaker for the current minimum phase. The magnetic pressure associated with the polar coronal holes seems to determine the acceleration of the high-speed wind. The weak field may be due to the fact that the polar field has not fully developed after the field reversal around the year 2000 (Figure 1b). 


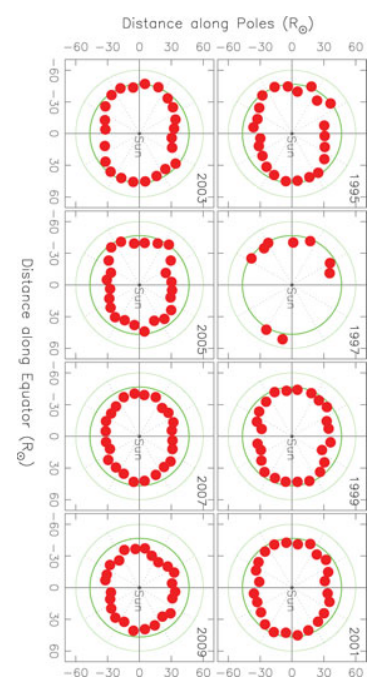

Figure 2. Shape of contours of constant density turbulence in the solar wind.

\subsection{Density-Turbulence Structures}

The drifting of density structures from high to low latitudes, seen in Figure 1a (bottom), is caused by the slow and gradual movement of concentrated magnetic field regions of the corona. It is likely due to the migration of small/medium-size coronal holes from polar to low latitude regions and the high-speed wind from these coronal holes interacting with the low-speed wind, causing compression in front of the high-speed stream. The latitudinal spread of density patterns is also consistent with the HCS tilt angle (Figure 1b), which is maximum at the time of polarity reversal of the cycle and during which a large number of coronal mass ejections (CMEs) dominate the heliosphere (e.g., Yashiro et al. 2004).

The density plot is also consistent with the solar wind disappearance period, around mid 1999 (low level of density turbulence), and co-rotating interaction regions (CIRs) dominating the heliosphere in the first half of 2003 (intense density turbulence along the latitudinal direction). It may be noted that during late October and early November 2003, a number of CME events prevailed in the interplanetary medium. However, the effect of a CME over the Sun-Earth distance is limited to 2-4 days after its onset and weakens with solar distance. Whereas, in the case of CIR events, the influence of each event is seen for several days, with a systematic increase in density turbulence with radial distance. Moreover, they show a latitudinal pattern. For CIR events during 2003, the latitudinal distribution and the radial evolution of the enhancement of turbulence have been observed (Manoharan 2008). The CIR-dominated period, in the first half of 2003, is in agreement with the moderate-to-severe storms observed at the Earth (Figure $1 \mathrm{~b}$, refer to $\mathrm{Kp}$ index plot). As observed in the speed plot, the latitudinal extents of low-turbulence regions at the poles also show remarkable changes between the current and previous minimum phases. The average level of turbulence at the current minimum seems to be considerably lower than that of the previous cycle.

\subsection{Scattering Diameter of the Corona}

Figure 2 displays constant $\delta N_{e}(R)$ plots, at different phases of the solar cycle. The plot for the year 1997 has been limited by weak-scattering observations and the last plot includes data up to May 2009. In general, a given level of turbulence is observed closer 
to the Sun at the poles than at the equator. However, depending on the phase of the solar cycle, the diameter of the contour can vary along the poles, but, remains nearly the same along the equator (e.g., Manoharan 1993).

The important point to note in this analysis is that after the year 2003, the overall diameter of the $\delta N_{e}(R)$ contour has gradually decreased with respect to the Sun's center. In other words, the same level of turbulence seems to move closer to the Sun. Thus, the radial dependence of turbulence $\left(C_{N_{e}}^{2}(R) \sim\left[\delta N_{e}(R)\right]^{2}\right.$, which typically varies as $\left.R^{-4}\right)$ suggests that the scattering diameter of the corona has gradually shrunk towards the Sun. In other words, the scattering power $\left(C_{N_{e}}^{2}(R)\right)$ remained nearly the same at all latitudes between 1989 and 2003, but decreased $\sim 50 \%$ around middle of 2009 at low-latitudes.

\section{Discussion and Conclusions}

The present large-scale 3-D features of solar wind speed and density turbulence are remarkably different from that of the previous cycle. In the current minimum phase, the extent of the low-speed region along the equatorial belt is considerably wider than that of the previous cycle; whereas the high-speed regions have shrunk towards the poles in contrast to the low-latitude extent of the previous cycle. The other important result of this study is that after the year 2003, the overall scattering diameter of the corona has gradually decreased with respect to the Sun's center. These results are consistent with the ecliptic and off-ecliptic studies (e.g., Lee et al. 2009; McComas et al. 2008; Smith \& Balogh 2008; Tokumaru et al. 2009).

The weak fields observed at the poles, as well as corresponding solar wind speed and density turbulence for the current low activity, are possibly caused by the changes in the movement of large-scale fields, as the reversal of polarity progresses. It is linked to the rate of poleward and equatorward meridional flows, which transport the unbalanced magnetic flux (e.g., Sheeley 2008). Moreover, the flux-transport dynamo has predicted weak polar fields and a long solar cycle (e.g., Choudhuri et al. 2007).

\section{Acknowledgements}

I thank all the members of the Radio Astronomy Centre for making the Ooty Radio Telescope available for IPS observations. I also thank the National Space Science Data Center for OMNI data and the Wilcox Solar Observatory for the magnetic field data. This work is partially supported by the CAWSES-India Program, which is sponsored by ISRO.

\section{References}

Choudhuri, A. R., Chatterjee, P., \& Jiang, J. 2007, Phy. Rev. Lett., 98, 131103

Lee, C. O. et al. 2009, Solar Physics, 256, 345

Manoharan, P. K. 1993, Solar Physics, 148, 153

Manoharan, P. K. et al. 2000, ApJ, 530, 1061

Manoharan, P. K. 2006, Solar Physics, 235, 345

Manoharan, P. K. 2008, in B. N. Dwivedi \& U. Narain (eds.), Physics of the Sun and its Atmosphere, (World Scientific, Singapore), p235-266

McComas, D. J. et al. 2008, Geophys. Res. Lett., 35, 18103

Sheeley, Jr., N. R. 2008, ApJ, 680, 1553

Smith, E. J. \& Balogh, A. 2008, Geophys. Res. Lett., 35, L22103

Swarup, G. et al. 1971, Nature Phys. Sci., 230, 185

Tokumaru, M. et al. 2009, Geophys. Res. Lett., 36, L091001

Yashiro, S. et al. 2004, J. Goephys. Res., 109, 7105 